\title{
Exclamatives échoïques : rendement fonctionnel et réalisations prosodiques
}

\author{
Gilles Corminboeuf \& Frédéric Gachet \\ Universités de Bâle et de Fribourg \\ Projet FNS n ${ }^{100012-146773}$ \\ gilles.corminbouef@unifr.ch, frederic.gachet@unifr.ch
}

\begin{abstract}
Résumé. Notre étude porte sur des énoncés à valeur exclamative comme « Moi, me railler de vous ? » dans cet extrait de Dom Juan :

Charlotte. - Monsieur, cela vous plaît à dire, et je ne sais pas si c'est pour vous railler de moi.

Dom Juan. - Moi, me railler de vous ? Dieu m'en garde ! Je vous aime trop pour cela, et c'est du fond du coeur que je vous parle. (Molière, Dom Juan, 1673)

La construction est formée d'un pronom tonique («Moi ») suivi d'un groupe verbal organisé autour d'un infinitif («me railler de vous ») et d'une marque modale (ici, le point d'interrogation). Elle est facultativement accompagnée d'un segment à valeur réfutative (ici, «Dieu m'en garde !»). Nous proposons une description sémantique et prosodique de ces exclamatives peu étudiées dans la littérature scientifique. Nous verrons dans un premier temps que la construction consiste en un schème syntaxique qui peut s'actualiser de différentes manières. Cette variabilité, en plus de questionner l'équivalence interprétative entre les différentes formes, pose des problèmes de description dans la mesure où on peut se demander si les formes observées ressortissent à un seul ou à plusieurs patrons syntaxiques. Nous détaillerons ensuite les rendements sémantico-pragmatiques de la construction, en particulier son caractère polyphonique, son orientation négative et sa nature exclamative. Dans la dernière partie de l'étude, nous étudierons - à partir d'enregistrements de pièces de théâtre - les réalisations prosodiques possibles de la construction, telle qu'elle est déclamée par des acteurs de théâtre. Nous relierons les contours intonatifs repérés à diverses visées pragmatiques. Nos données sont issues de divers corpus, en particulier des œuvres théâtrales de la base Frantext. Le discours théâtral est en effet particulièrement pourvoyeur de cette construction.
\end{abstract}

\begin{abstract}
Our paper presents a semantic and prosodic analysis of exclamative utterances such as "Moi, me railler de vous ? " in this excerpt of Molière's Dom Juan :

Charlotte. - Monsieur, cela vous plaît à dire, et je ne sais pas si c'est pour vous railler de moi. Dom Juan. - Moi, me railler de vous ? Dieu m'en garde ! Je vous aime trop pour cela, et c'est du fond du coeur que je vous parle. (Molière, Dom Juan, 1673)

This structure, better known in English as «Mad Magazine sentence » («What, me worry ? »; see Akmajian 1984), is made up of a disjunctive pronoun and an infinitive verb phrase. It is optionally followed by a refutative segment («Dieu m'en garde!» in the excerpt above). We firstly show that our structure consists in a syntactic pattern that can be realised in several ways. Our paper deals then with the pragmatic effects of the structure, its polyphonic nature, its negative orientation and its exclamative value. In the last part of the paper, we study prosodic properties of the structure when performed by stage actors. We attempt to relate the prosodic realisations of the structure with its pragmatic purposes. Since our structure is very frequently used in theatre repertoire, our data mostly comes from plays from the French literature database Frantext.
\end{abstract}




\section{Introduction}

Les phénomènes échoïques - qui relèvent de la polyphonie linguistique (Ducrot 1984) - sont particulièrement bien représentés dans le genre théâtral :

(1) Monsieur Remy. - Hum ! Quoi ? Entendez-vous ce que je vous dis, qu'elle a quinze mille livres de rente ? Entendez-vous ?

DORANTE. - Oui, Monsieur ; mais en eût-elle vingt fois davantage, je ne l'épouserais pas ; nous ne serions heureux ni l'un ni l'autre : j'ai le cœur pris ; j’aime ailleurs.

MONSIEUR REMY, d'un ton railleur, et traînant ses mots. - J'ai le cœur pris : voilà qui est fâcheux ! Ah, ah, le cœur est admirable ! Je n’aurais jamais deviné la beauté des scrupules de ce cœur-là, qui veut qu'on reste intendant de la maison d'autrui pendant qu'on peut l'être de la sienne ! Est-ce là votre dernier mot, berger fidèle ? (Marivaux, Les Fausses Confidences, 1738)

(2) DORANTE. - Répondez donc ; je ne demande pas mieux que de me tromper. Mais que disje ? Mario vous aime.

SILVIA. - Cela est vrai.

DORANTE. - Vous êtes sensible à son amour, je l'ai vu par l'extrême envie que vous aviez tantôt que je m'en allasse ; ainsi vous ne sauriez m'aimer.

SILVIA. - Je suis sensible à son amour ! qui est-ce qui vous l'a dit? Je ne saurais vous aimer ! qu'en savez-vous? Vous décidez bien vite. (Marivaux, Le Jeu de l'amour et du hasard, 1730)

(3) ToinetTe. - (...) Mais, monsieur, mettez la main à la conscience ; est-ce que vous êtes malade ?

ARGAN. - Comment, coquine, si je suis malade ! Si je suis malade, impudente ! (Molière, Le Malade imaginaire, 1673)

Dans (1), la didascalie d'un ton railleur, et traînant ses mots dénonce la mention qui suit (J'ai le cœur pris) : M. Rémy reprend verbatim une partie du discours de Dorante afin de s'en gausser. Dans (2), la mention du discours de Dorante dans la réplique de Silvia s'accompagne d'une transposition des pronoms personnels; les questions rhétoriques partielles qui suivent ces segments visent, tout comme le phénomène de mention, à disqualifier ce discours, - ou du moins à remettre en question son bien-fondé. Le segment souligné dans l'extrait (3) fait écho, au moyen d'une interrogative indirecte tronquée, à la question de Toinette, de manière à lui dénier tout à-propos ( $c f$. également les jugements de valeur coquine et impudente).

Nous consacrons cette étude à exposer les résultats d'une recherche sur des séquences échoïques aux propriétés bien particulières. La plus patente d'entre elles est que l'écho est produit au moyen d'une construction syntaxique dédiée à cette fonction singulière. En voici un exemple :

(4) MEDEE. - (...) Je reprends cette foi que tu crains d'accepter,

Et préviens un ingrat qui cherche à me quitter.

JASON. - Moi, vous quitter, Madame ! ah ! que c’est mal connoître

Le pouvoir du beau feu que vos yeux ont fait naître ! (Corneille, La Toison d'or, 1682)

La structure soulignée dans (4) est formée d'un pronom tonique suivi d'un infinitif et d'une marque modale (le point d'exclamation dans 4). Elle est facultativement accompagnée d'un segment à valeur de dénégation (que c'est mal connoître le pouvoir du beau feu que vos yeux ont fait naître ! dans 4). L'exemple (4) est relativement prototypique, mais il faut mentionner diverses variantes de ce schème syntaxique. Ainsi, le pronom tonique peut être placé après l'infinitif (Me pendre, moi !), être à une autre personne que la première (Lui, me croire coupable !), être remplacé par un SN indéfini (Un sage être amoureux !), etc. (v. Corminboeuf \& Gachet, soumis)

Nos données sont issues de la base Frantext, de lectures diverses (œuvres littéraires, presse écrite, bandes dessinées, web) et d'exemples oraux recueillis à la volée. Nous avons réuni environ 200 occurrences de 
cette construction à l'infinitif - dont la moitié ressortit au genre théâtral ${ }^{1}$-, ainsi qu'une centaine d'occurrences de structures apparentées diverses (v. § 2). Le genre interactif du discours théâtral est particulièrement pourvoyeur de cette construction, qui semble donc sensible au type de discours.

Dans un premier temps, nous montrerons que cette construction consiste en un schème syntaxique qui peut s’actualiser de différentes manières (§ 2). Une telle variabilité questionne l'équivalence interprétative entre les différentes réalisations ; on peut également se demander si ces actualisations ressortissent à un seul ou à plusieurs patrons syntaxiques. Nous détaillerons ensuite les propriétés pragmatiques de la construction, en particulier son caractère réactif, son orientation négative et sa nature exclamative (§ 3 ). Dans un troisième temps, nous étudierons - dans des enregistrements de pièces de théâtre - comment les acteurs déclament cette construction. Nous avons repéré trois principaux contours intonatifs, qu'on peut relier à autant de visées pragmatiques (§ 4).

\section{Variations formelles du schème}

La composante prédicative de la construction peut prendre la forme d'un infinitif (4), mais également être constituée d'une forme verbale au subjonctif (5) ou au conditionnel $(6)^{2}$ :

(5) IDOMENEE. - Oui, mon fils : mon coeur reconnoissant

Ne veut point que ma mort vous en fasse un présent.

Je sais que c'est un rang que votre coeur dédaigne ;

Mais qu'importe ? Il le faut... régnez...

IDAMANTE. - Moi, que je règne,

Et que j'ose à vos yeux me placer dans un rang

Où je dois vous défendre au prix de tout mon sang !

À cet ordre, seigneur, est-ce à moi de souscrire ?

Ciel ! est-ce à votre fils à vous ravir l'empire ? (Crébillon, Idoménée, 1706)

Le point commun entre les actualisations infinitive et subjonctive du schème est qu'elles permettent de viser un effet généralisant. La construction transforme un fait énoncé préalablement (et tenu pour vrai) auquel elle fait écho - en une simple éventualité.

Le conditionnel se comporte un peu différemment du subjonctif et de l'indicatif, dans la mesure où il présente d'emblée le fait comme une hypothèse :

(6) HERMIONE. - Hé quoi ? votre haine chancelle ?

Ah ! courez, et craignez que je ne vous rappelle.

N'alléguez point des droits que je veux oublier ;

Et ce n'est pas à vous à le justifier.

ORESTE. - Moi, je l'excuserois ? Ah ! vos bontés, Madame,

Ont gravé trop avant ses crimes dans mon âme. (Racine, Andromaque, 1668)

En exprimant une surprise suscitée par une hypothèse (le fait qu'Oreste excuse Pyrrhus dans 6), la structure vise à nier que ce fait soit possible.

Dans une autre variante, le composant prédicatif peut n'être constitué que d'un adjectif (7) ou d'un syntagme nominal (8) :

(7) ARLEQUin, entendant nommer sa maîtresse. - Monsieur, ne me parlez plus d'elle ; car, voyez-vous, j'ai dans mon esprit qu'elle est amoureuse, et j'enrage.

LELIO. - Amoureuse ! elle amoureuse ? (Marivaux, La Surprise de l'amour, 1723)

(8) Trivelin. - Avant que de me réduire tout à fait à cet état humiliant, je commençai par vendre ma garde-robe.

FRONTIN. - Toi, une garde-robe ! (Marivaux, La Dispute, 1744) 
De notre point de vue, ces différents formats - sans être des équivalents sémantiques à proprement parler - ne révèlent pas de différence interprétative notable. Cette actualisation différenciée d'un même (?) schème formel, couplé à un rendement fonctionnel relativement stable pourrait légitimer une approche « constructionnelle » (v. à ce propos Fillmore \& al. 1988, Lambrecht 1990, Bouveret \& Legallois 2012, Corminboeuf \& Gachet, soumis) Dans le cadre de cette étude, nous laisserons cette discussion de côté.

A notre sens, l'utilisation de l'indicatif est, elle, associée à un fonctionnement sensiblement différent :

(9) AGNEs. - Le lendemain, étant sur notre porte,

Une vieille m'aborde, en parlant de la sorte :

«(...) Et vous devez savoir que vous avez blessé

Un cœur qui de s'en plaindre est aujourd'hui forcé. »

ARNOLPHE, à part. - Ah ! suppôt de Satan ! exécrable damnée !

AGNES. - « Moi, j’ai blessé quelqu’un ! fis-je toute étonnée.

- Oui, dit-elle, blessé, mais blessé tout de bon ; (...) » (Molière, L’École des femmes, 1663)

Dans (9), comme dans les exemples précédents, la formule (Moi, j’ai blessé quelqu'un !) reprend un fait mentionné dans le contexte antérieur (i.e. présenté comme avéré). La différence est qu'Agnès met en doute le fait d'avoir blessé quelqu'un au moyen d'une "simple » demande de confirmation, sans provoquer l'effet généralisant de l'infinitif. En effet, dans notre construction, l'infinitif n’exprime pas la surprise à propos d'un fait, mais bien à propos de l'éventualité que ce fait puisse avoir lieu. Avec Moi, blesser quelqu'un! Agnès aurait remis en question la possibilité qu'elle ait pu blesser, autrement dit la compatibilité entre moi et l'action de blesser autrui ${ }^{3}$. Le choix de l'infinitif, tout comme celui du subjonctif ou du conditionnel, n’est donc pas anodin : c'est celui d’un effet généralisant, que ne permet pas l'indicatif. Cette analyse nous semble conciliable avec l'observation d'Akmajian (1984) qui parlait d’ « irrealis interpretation » à propos du fait dénoté dans la construction.

\section{Rendement pragmatique}

\subsection{Une construction échoïque}

La construction s'affiche comme réactive : elle réinvestit en effet sous forme de mention un fait (présenté comme) valide dans la mémoire discursive ${ }^{4}$. La reprise en écho peut être plus ou moins fidèle :

(10) Angelique. - (...) Ne m’abandonne point, je te prie, dans l'extrémité où je suis.

TOINETTE. - Moi, vous abandonner ? j'aimerois mieux mourir. (...) (Molière, Le Malade imaginaire, 1673)

(11) EURYALE. - (...) Je te promets ici de dire que l'amour

M’a rangé sous ses lois, et me brave à son tour,

Et je consens encor que tu me fasses honte

Des foiblesses d'un cœur qui souffre qu'on le dompte.

ARBATE. - Moi, vous blâmer, Seigneur, des tendres mouvements

Où je vois qu'aujourd'hui penchent vos sentiments ! (Molière, La Princesse d'Élide, 1664)

Dans (10), Moi, vous abandonner ? reprend presque à la lettre le Ne m'abandonne point de la réplique précédente, alors que dans (11), Arbate reformule intégralement les propos d'Euryale. Fondamentalement polyphonique, la construction met en jeu le point de vue d'un autre énonciateur, le plus souvent l'allocutaire. La stratégie consistant à exhiber le discours ou le point de vue d'autrui n'est pas sans conséquence, on le verra infra (§ 3.3). 


\subsection{Une demande de confirmation de dicto}

Au plan formel, la construction se présente comme une sorte de demande de confirmation de dicto (Fontaney 1991). Autrement dit, on fait comme si on avait mal entendu, mal interprété, et qu'on demandait de répéter afin de confirmer ce qui vient d'être dit. C'est ce type de comportement qu'adopte Angélique dans l'extrait (12) :

(12) Monsieur De Sotenville. - Allons, venez, ma fille, que votre mari vous demande pardon. Angelique, descendue. - Moi ? lui pardonner tout ce qu'il m'a dit ? Non, non, mon père, il m'est impossible de m’y résoudre, et je vous prie de me séparer d’un mari avec lequel je ne saurois plus vivre. (Molière, George Dandin, 1669)

Littéralement, Angélique demande à son père de confirmer si c’est vraiment à elle qu'il s’adresse et s’il lui enjoint bien de pardonner à son mari. Par ce moyen détourné, elle gratifie la requête paternelle d'une cinglante fin de non-recevoir. En effet, la formulation Moi ? lui pardonner tout ce qu'il m'a dit ? sousentend <il est exclu que je lui pardonne>, ce que confirme la suite de sa réplique (Non, non...). Cette attitude de rejet s'explique par le fait que moi et lui pardonner sont pour elle incompatibles. A noter que dans le discours théâtral et en particulier avec le tonique de première personne (moi), la feinte qui consiste à faire semblant d'avoir mal entendu s'accompagne volontiers d'une tendance à surjouer une réaction de surprise aux paroles qui précèdent.

\subsection{Une orientation négative}

Comme on le voit dans l'extrait précédent, cette demande de confirmation feinte invite à calculer une affirmation négative implicite ${ }^{5}$. L'étonnement surjoué à propos de la compatibilité entre les deux éléments de la construction est une manière indirecte de nier cette compatibilité. De manière facultative, cette négation implicite peut être verbalisée à la suite de la construction à titre de "réponse » autolocutée, au moyen de formes du genre jamais, non, impossible, plutôt mourir, Dieu m'en garde, en aucun cas, pour qui me prend-on ? etc ${ }^{6}$. Cela rappelle bien sûr l'orientation négative des questions oratoires, dont une occurrence (Et l'amour a-t-il été fait pour des gens bâtis comme lui ?) est formulée peu après notre construction dans l'exemple (13) :

(13) CLEANTE. - Comment va notre affaire ? Les choses pressent plus que jamais ; et depuis que je ne t'ai vu, j'ai découvert que mon père est mon rival.

LA FLECHE. - Votre père amoureux ?

CLEANTE. - Oui ; et j'ai eu toutes les peines du monde à lui cacher le trouble où cette nouvelle m'a mis.

LA FLECHE. - Lui se mêler d'aimer ! De quoi diable s'avise-t-il ? Se moque-t-il du monde? Et l'amour a-t-il été fait pour des gens bâtis comme lui ? (Molière, L'Avare, 1668)

Dans l'extrait suivant, le connecteur au contraire enchaîne clairement sur cet implicite négatif :

(14) SopHIE. - Après ce que Madame a dû vous confier,

Votre dessein n'est plus de me sacrifier.

ARGANT. - Moi, te sacrifier ! quand je veux au contraire

Te donner pour époux quelqu'un qui t’a sçu plaire ; (La Chaussée, Le Préjugé à la mode, 1735)

La forme verbale au conditionnel (je le voudrais) se présente dans (15) comme une réponse auto-locutée à la demande qui précède, invitant à calculer le même implicite négatif (<je ne peux pas l'aimer $>$ ) :

(15) LUCINDE, tendrement. - Vous n'aimez donc point Cydalise, Lindor ?

LINDOR. - Moi ? L’aimer ! Je le voudrais, ingrate. Ma vengeance en serait plus complète : mais est-on le maître de disposer si facilement d'un coeur où vous avez régné ? (...) (Palissot de Montenoy, Le Cercle, 1755) 
Le rendement pragmatique global de notre construction est celui d'une réaction incrédule à l'association des deux membres de la construction, avec une valeur de rejet.

\subsection{Une construction exclamative}

En plus du point d'exclamation qui conclut majoritairement notre construction (concurrencé seulement par le point d'interrogation), on observe communément dans son environnement des morphèmes et locutions exclamatives (ah ! oh !, Quoi !, Juste ciel !). Ce voisinage fournit déjà un indice pour considérer que la construction est exclamative, mais le fonctionnement même de la structure donne également des arguments en faveur d'un statut intrinsèquement exclamatif :

(16) CHARLOTTE. - Monsieur, cela vous plaît à dire, et je ne sais pas si c'est pour vous railler de moi.

DOM JuAn. - Moi, me railler de vous ? Dieu m'en garde ! Je vous aime trop pour cela, et c'est du fond du coeur que je vous parle. (Molière, Dom Juan, 1673)

Dans (16), Don Juan pourrait réagir au soupçon de Charlotte par un démenti explicite : non, pas du tout. Une telle réaction ne serait pas intrinsèquement exclamative. En revanche, la formulation qu'il emploie (Moi, me railler de vous ?) a en commun avec bon nombre de structures exclamatives le fait d'exprimer de manière implicite qu'un fait excède les normes habituelles (Gachet \& Corminboeuf 2012). Plus précisément, elle invite à inférer que l'apparentement entre ce que décrivent ses deux membres dépasse, pour son locuteur, les limites du concevable. En l'occurrence, dans (16), la formule de Don Juan vise à faire comprendre implicitement et de manière expressive qu'il est impensable pour lui d'être associé au fait de se moquer de la paysanne. C'est en cela que la construction peut être considérée comme fondamentalement exclamative ; le rejet d'un apparentement inenvisageable (entre moi et me railler de vous) est implicitement « montré », et non explicitement verbalisé.

Nous avons postulé supra que la construction fonctionne comme une sous-classe de demandes de confirmation de dicto ayant ritualisé une inférence négative (le rejet exclamatif de la compatibilité entre ce qui est exprimé par ses membres). Cette inférence semble se superposer à la valeur initiale de demande de confirmation (et non s'y substituer). Même si l'inférence est fortement ritualisée, l'orientation négative est sous-entendue et il est toujours possible de l'annuler. L'allocutaire peut ainsi faire mine de ne pas la reconnaître et traiter la construction comme une simple demande de confirmation. Autrement dit, la valeur "première » de demande de confirmation n'est jamais totalement absente de la construction (Corminboeuf \& Gachet, soumis).

En résumé, la construction du type Moi, me railler de vous ? est à la base un type particulier de demande de confirmation, qui a ritualisé une inférence de type négatif servant exprimer de manière exclamative le rejet de la compatibilité entre ce qui est exprimé par ses deux membres.

Dans la section 4, nous nous demanderons si les réalisations prosodiques observées dans notre corpus d'oral «théâtral » corroborent notre description sémantico-pragmatique, en particulier la nature exclamative de la structure et l’orientation négative qui lui est attachée.

\section{Les réalisations prosodiques de la construction}

A l'écrit, notre construction porte systématiquement une marque modale finale : un point d'exclamation ou un point d'interrogation. Mais ce fait de ponctuation, très labile en particulier dans les textes des XVII ${ }^{\mathrm{e}}$ et XVIII ${ }^{\mathrm{e}}$ siècles, nous en apprend peu sur la construction. Un examen des possibilités prosodiques de notre structure nous a donc semblé utile à améliorer sa connaissance. Il se heurte cependant à des difficultés pratiques. Il est en effet difficile de réunir une collection d'extraits oraux de cette construction, principalement pour deux raisons. D’un côté, les situations d'interview (récits de vie, etc.) qui composent la plus grande partie des corpus oraux ne sont pas un terrain favorable à la présence massive d'expressions exclamatives, encore moins à valeur polémique. D’autre part, comment formuler une 
recherche automatique qui permettrait de ne pas manquer les rares occurrences éventuellement présentes?

Au cours de nos recherches, nous avons pourtant pu attraper à la volée (sans pouvoir les enregistrer) l'une ou l'autre occurrence attestant que la structure est bien vivante dans le français parlé, et pas seulement dans la littérature. La première avait comme contexte une réunion informelle entre linguistes. L1 y évoquait les rapports entre un collaborateur scientifique (nommé par il) et les étudiants qu’il encadrait :

(17) L1 : - j'en revenais pas + il a HURlé sur les étudiants L2 : - lui hurler (oral, à la volée, 12 mars 2015) ${ }^{7}$

Dans (17), la construction utilisée par L2 réagit au caractère excessif de l'intervention de L1. Il était manifeste pour les personnes présentes dans ce contexte que hurler était un verbe inapproprié à la personne désignée par il, notoirement peu portée à élever la voix. La réaction d’étonnement de L2 est ici sincère et spontanée, et non feinte ou surjouée comme on a pu l'observer dans certains des exemples précédents ${ }^{8}$.

La deuxième occurrence a été produite lors d'une discussion entre quatre adolescents regagnant leur domicile en train après une journée de classe. La teneur générale très relâchée des propos qui ont précédé et suivi l'occurrence prouve si besoin était que la structure est productive dans un registre de langue très éloigné du littéraire :

(18) L1 : - à Romont je connais la salle de gym

L2 : - toi faire du sport (oral, à la volée, 7 novembre 2015)

Ayant été tous deux recueillis à la volée, ces exemples sont malheureusement peu en mesure de nous renseigner sur la prosodie de la construction.

\subsection{Avertissement et méthodologie}

Pour les raisons évoquées supra, il ne nous a pas été possible d’établir une collection de données orales spontanées de notre construction. Plutôt que de renoncer à notre étude prosodique, nous avons toutefois pensé pouvoir user d’un expédient. Comme la construction est très bien représentée dans les textes de théâtre, nous avons opté pour une analyse prosodique basée sur la déclamation des acteurs. Puisqu'il s'agit d'étudier la façon dont est intonée par un acteur du $\mathrm{XX}^{\mathrm{e}}$ ou du XXI ${ }^{\mathrm{e}}$ siècle une construction sise dans des textes des XVII ${ }^{\mathrm{e}}$, XVIII ${ }^{\mathrm{e}}$ et XIX ${ }^{\mathrm{e}}$ siècles, il faut bien sûr relativiser les résultats obtenus, tenir compte du fait que les réalisations observées ne relèvent pas de l'oral spontané, mais sont des simulacres. La déclamation des acteurs ne peut naturellement pas être assimilée à de l'oral spontané, étant d'abord dictée par la réalisation d'un texte écrit (et ponctué), de plus travaillée et retravaillée, motivée par une recherche d'effets qui peuvent être plus ou moins naturels ou artificiels. Toutefois, on peut présumer que l'emploi des contours intonatifs et autres paramètres prosodiques n’y est pas incompatible avec les lois qui président à la communication orale... Aussi travaillée et chargée d'artifice que puisse être leur déclamation, les acteurs cherchent en principe à se faire comprendre, en se servant et en jouant d'un « code » prosodique qu'ils partagent avec leur public, faute de quoi ils encourent le risque de rendre opaque le texte qu'ils veulent transmettre. Tout bien pesé, il nous a donc paru possible de nous servir d'un corpus composé d'extraits de représentations théâtrales pour examiner, avec toute la prudence requise, la prosodie de nos structures. Cette option nous a semblé la seule manière d'approcher un tant soit peu la prosodie de cette construction. Malgré qu'il y ait « du jeu » (dans les deux sens du terme !) entre les réalisations prosodiques de l’oral non planifié et la prosodie représentée sur une scène de théâtre - nous pensons que le... jeu en vaut la chandelle.

Les exemples de notre corpus prosodique sont issus de représentations de pièces de Molière (Tartuffe, Dom Juan, le Misanthrope, George Dandin, L'Avare) et Racine (Phèdre, Andromaque, Bérénice) pour le $\mathrm{XVII}^{\mathrm{e}}$ siècle, ainsi que, pour les deux siècles suivants, de la Surprise de l'amour (Marivaux), de Ruy Blas (V. Hugo) et Cyrano de Bergerac (E. Rostand). Les acteurs célèbres y côtoient des artistes plus obscurs 
(amateurs, jeunes comédiens passant un examen d'art dramatique, particuliers postant une lecture sur youtube ${ }^{9}$ ).

Le corpus comporte une cinquantaine d'exemples ${ }^{10}$. Nous nous sommes limités aux occurrences mettant en jeu un pronom tonique suivi d'un infinitif. Pour certaines répliques, nous avons pu trouver plusieurs enregistrements différents, permettant de comparer diverses réalisations prosodiques d'une même séquence. La méthode de constitution et la taille de notre corpus le rendent impropre à toute exploitation quantitative ou statistique, nous nous bornons donc à une observation qualitative, en y cherchant surtout des informations générales sur les possibilités prosodiques caractérisant nos structures. Nous essayons d'associer les réalisations prosodiques avec les effets communicatifs ressentis.

\subsection{Analyse du corpus prosodique}

Dans les études antérieures ayant trait aux constructions qui nous intéressent, on trouve des remarques prosodiques concernant deux types de caractéristiques : la manière de segmenter et l’intonation.

Concernant la nature segmentée ou non de la construction, Wenger (2009 : §2.2.2.) suggère de distinguer « unified intonation contour » et « comma intonation », considérant que « speaker judgements differ as to whether ARIs form one intonation phrase, or rather two distinct ones ». A l'écrit, la ponctuation semble pouvoir se faire le reflet de plusieurs possibilités de segmentation. On observe entre autres que la variante introduite par un syntagme nominal indéfini est le plus souvent ponctuée en un seul tenant :

(19) SOLIMAN, fumant par intervalles. - Je ne sors point de mon étonnement ;

Une esclave parler avec cette arrogance ! (Il fume.) (...) (Favart, Les Trois Sultanes, ou Soliman II, 1762)

D’autres typographies invitent à oraliser la construction comme une suite de deux demandes de confirmation :

(20) Bianca CAStafiore. - (...) C’est trop drôle !... Ha ! Ha ! Ha ! Vous devez bien rire, n’estce pas, messieurs.

DUPOND. - Rire, madame ?... Nous, madame ?... Vous voulez rire, madame !!!... Nous vous saluons, madame !... (Hergé, Les Bijoux de la Castafiore)

Dans (20), les vocatifs postposés aux éléments constitutifs de la construction indiquent que la séquence en gras est formée de deux périodes interrogatives successives.

Une question est de savoir s'il est envisageable de tirer quelque chose des signifiants prosodiques pour l'analyse syntaxique. De notre point de vue, la construction s'analyse en général comme une période binaire, formée de deux demandes de confirmation successives. Ces deux interrogatives sont syntaxiquement autonomes mais, réunies au sein d'une même construction, elles fonctionnent comme une séquence exclamative. Des extraits comme (19) et (20) conduisent cependant à envisager la possibilité que certaines occurrences fonctionnent comme des périodes unaires (19), ou comme une suite de deux périodes intonatives distinctes (20).

Quant aux propriétés intonatives de notre construction, on trouve dans la littérature scientifique de rares hypothèses à leur propos. Jespersen (1924 : 174-176) analyse la construction dans une section intitulée « Les nexus qui expriment le rejet ». Selon l'auteur, « dans la langue parlée l'intonation [est] en anglais la même que l'interrogation, mais souvent exagérée et, dans bien des cas, appliquée séparément aux deux éléments du nexus ». Nous tenterons de confronter nos données de corpus à cette hypothèse.

\subsubsection{Segmentation}

Nous examinons d'abord les possibilités de segmentation prosodique de la structure. Dans notre corpus, on observe un continuum de réalisations que nous pouvons classer en trois catégories. Dans la première, le pronom tonique et le syntagme verbal à l'infinitif sont séparés par une frontière prosodique majeure, chacun constituant alors une unité prosodique majeure (UPM). La réalisation de l'exemple (21=16) par 
Michel Piccoli, dans le téléfilm de Marcel Bluwal (1965), en donne une illustration (v. Fig. 1) : l'acteur y insère une pause de 72 cs entre moi et me railler de vous.

(21) Moi, me railler de vous ? Dieu m'en garde ! (Molière, Dom Juan, 1673)

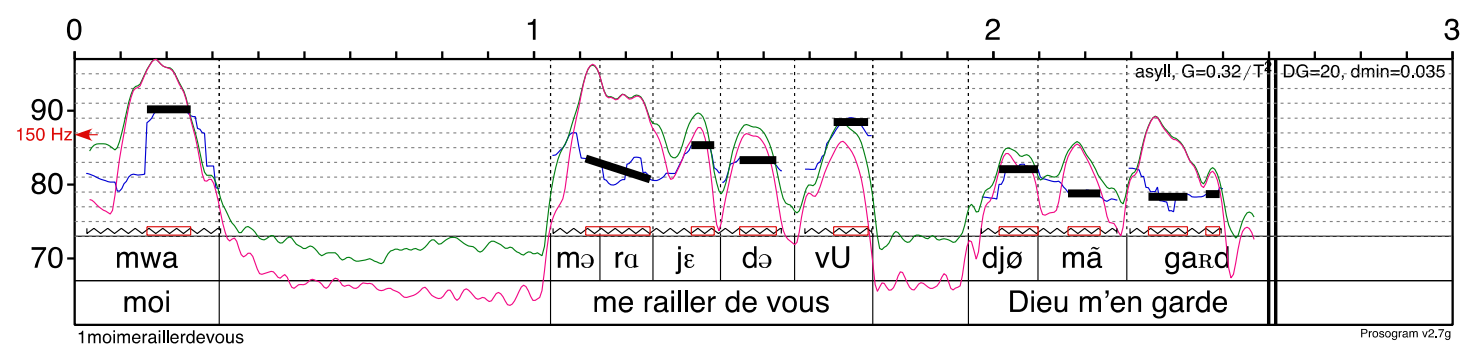

Figure 1 : prosogramme de l'exemple (21) (Michel Piccoli)

Ce type de réalisation fait de la structure une succession de deux interrogations autonomes, distinctes, autrement dit deux périodes interrogatives.

Un deuxième type de segmentation sépare le pronom et le syntagme verbal par une frontière prosodique plus faible, et en fait deux groupes intonatifs d'une même UPM. On peut l'illustrer avec une autre version de la réplique précédente (21), celle de Jean-Claude Durand dans la mise en scène d’Antoine Vitez en 1979 (Fig. 2) :

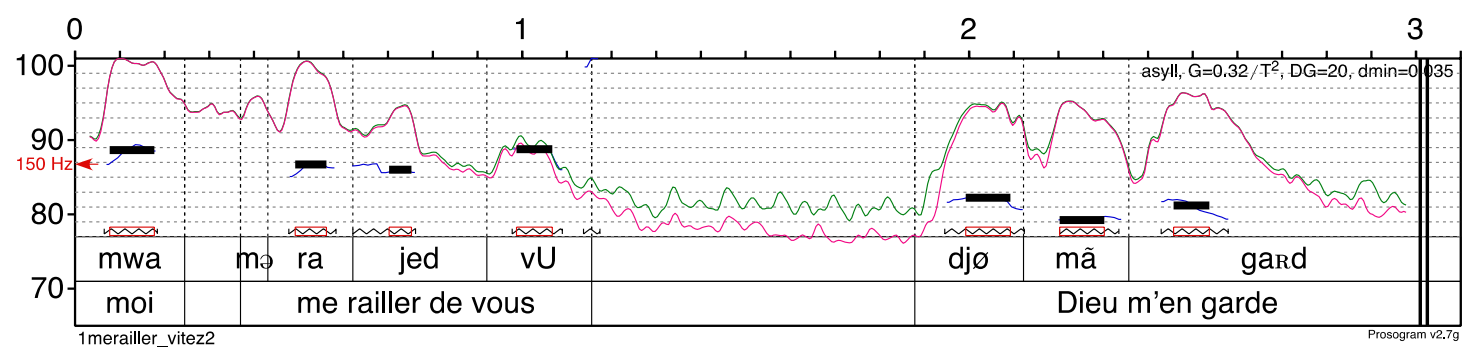

Figure 2 : prosogramme de l'exemple (21) (Jean-Claude Durand)

La structure est alors réalisée comme une période binaire, et ressentie grosso modo comme une construction segmentée.

A l'autre extrémité du continuum, la construction peut être réalisée comme un seul groupe intonatif. Le pronom n’est alors marqué que par une simple proéminence accentuelle, comme dans cette réalisation d’une réplique d'Angélique (22) tirée de George Dandin (v. Fig. 3) :

(22) George Dandin. - Je veux que vous y fassiez ce que fait une femme qui ne veut plaire qu'à son mari. Quoi qu'on en puisse dire, les galants n'obsèdent jamais que quand on le veut bien. Il y a un certain air doucereux qui les attire, ainsi que le miel fait les mouches ; et les honnêtes femmes ont des manières qui les savent chasser d'abord.

ANGELIQUE. - Moi, les chasser ? et par quelle raison ? (Molière, George Dandin, 1669)

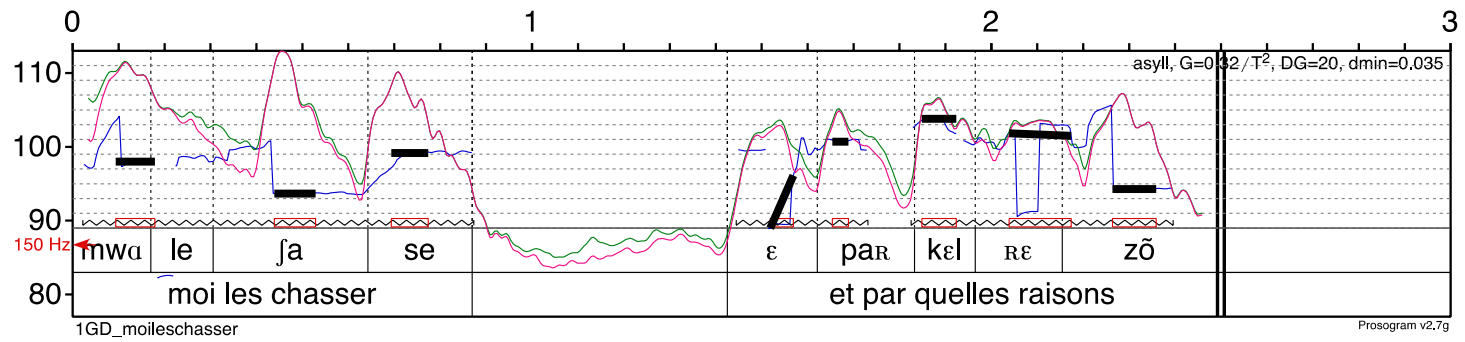

Figure 3 : prosogramme de l'exemple (22) 
Plus rarement, il arrive même que le pronom initial ne fasse l’objet d'aucune proéminence. C’est le cas dans la réplique suivante tirée de l'Orgon du Tartuffe (23), telle qu'elle est jouée par Jacques Charon dans sa mise en scène de 1973 pour la Comédie Française. Le pronom moi y est entièrement intégré dans le même groupe intonatif que la construction infinitive sortir de céans, le tout dans un débit très rapide (Fig. 3) :

(23) Monsieur LoyAL. - Monsieur, sans passion,

Ce n'est rien seulement qu'une sommation,

Un ordre de vider d'ici, vous, et les vôtres,

Mettre vos meubles hors, et faire place à d'autres,

Sans délai, ni remise, ainsi que besoin est...

ORGON. - Moi, sortir de céans ? (Molière, Le Tartuffe, 1669)

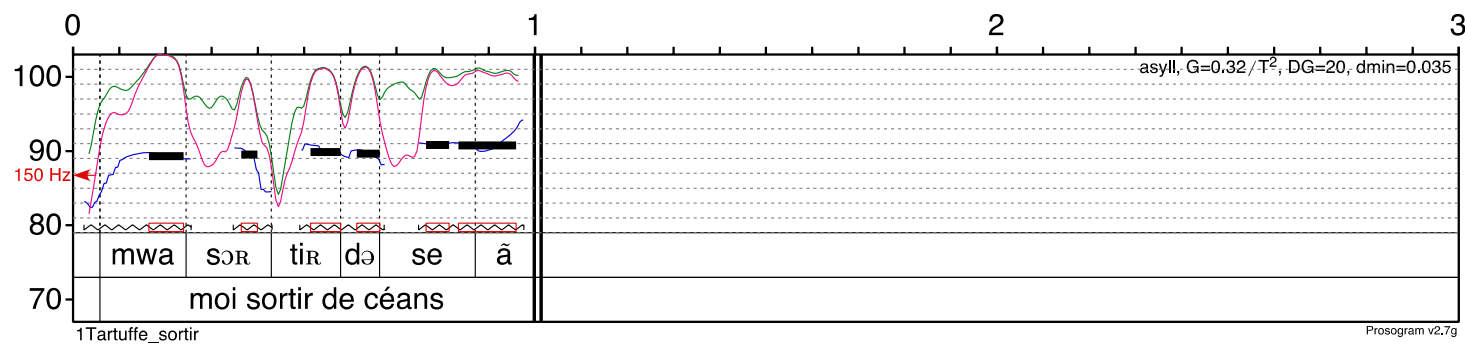

Figure 4 : prosogramme de l'exemple (23) (Jacques Charon)

Dans ces deux exemples, la construction est réalisée comme une seule énonciation, plus précisément comme une période unaire. Les locuteurs qui la prononcent de cette manière la traitent vraisemblablement comme une construction liée.

Il faut signaler que ces choix de segmentation semblent parfaitement indépendants de la ponctuation : celle-ci est identique dans les quatre exemples précédents, avec une virgule entre le pronom et le syntagme infinitif. Ces options de segmentation semblent bien confirmer l’idée que les locuteurs peuvent interpréter la construction comme deux périodes distinctes, comme une période binaire ou comme une période unaire.

Cela précisé, nous nous intéressons principalement aux contours intonatifs utilisés pour réaliser notre construction. Nous avons relevé trois différents cas de figure.

\subsubsection{Contour montant}

Dans le cadre de notre corpus, c’est avec un contour montant qu'est majoritairement réalisée notre structure. Il n'y a là rien d'étonnant, pour une construction qui se manifeste littéralement comme une demande de confirmation. Dans les cas où la structure est segmentée en deux UPM, il s'agit de deux interrogatives successives, comme dans la réalisation de (21) illustrée par la Fig. 1 supra, qui fait se succéder deux contours montants, sur moi et sur vous. L'exemple (22), qui fait au contraire de la structure moi les chasser un seul groupe intonatif à contour montant, la traite comme une seule demande de confirmation (v. Fig. 3 supra).

On observe dans le corpus une certaine variété dans l'amplitude des contours montants. Nous renonçons toutefois à déterminer à partir de quelle amplitude il faudrait considérer ces contours comme " exagérés » (cf. Jespersen 1924). Il nous paraît plus intéressant d'observer que les différentes amplitudes se prêtent à une gradation des effets expressifs. Les contours les plus amples semblent utilisés pour créer l'impression d'une réaction spontanée de surprise, et accentuer ainsi l'implicite réfutatif de la construction (le rejet de la compatibilité entre ce qui est exprimé par le pronom et par l’infinitif). 
A cet égard, nous pouvons tirer profit de nos deux réalisations de l'exemple (21) pour comparer deux attitudes prosodiques traduisant des partis pris d'interprétation différents de la part des acteurs. Dans cette réplique, Don Juan, qui tente de séduire la paysanne Charlotte, répond aux soupçons qu'elle formule quant à la sincérité de ses déclarations. Dans le film de Bluwal, le Don Juan campé par Michel Piccoli joue un étonnement sincère face à l’idée qu'il se moquerait de Charlotte. Il exhibe sa surprise au moyen de deux amples contours montants (v. Fig. 1 supra). Au contraire, dans la mise en scène de Vitez, JeanClaude Durand incarne un séducteur nettement plus distant, voire méprisant, qui semble s’opposer plus mollement, et avec davantage de hauteur, au soupçon de Charlotte. On peut observer dans la Fig. 2 supra qu'il marque la même séquence par un contour montant de moindre amplitude. Il faut bien sûr souligner que cette différence de comportement ne se traduit pas uniquement par l'amplitude mélodique. D’autres faits concourent à la confirmer, notamment la prosodie marquant l'énoncé qui suit notre structure (Dieu m'en garde) : là où Piccoli la marque d'un contour descendant nettement assertif, Durand lui applique une montée suspensive qui trahit, sinon son insincérité, du moins un engagement très modéré. Le comportement gestuel des acteurs donne une confirmation de plus : le Don Juan de Piccoli est tourné vers Charlotte et la regarde dans les yeux, tandis que celui de Durand dirige son regard vers la salle plutôt que vers la paysanne, à qui il tourne presque le dos.

\subsubsection{Contour « en cloche » (« intonation d'implication »)}

Dans d'autres exemples de notre corpus, la structure est réalisée intonativement avec un contour « en cloche ", autrement dit un contour montant-descendant sur la dernière syllabe. Ce contour correspond à ce que Delattre (1966) a baptisé l' « intonation d’implication ». Il s’applique généralement à des énonciations assertives, mais il est à noter qu'il sert également à marquer les demandes de confirmation, comme l'indique l'extrait suivant de la thèse que Portes (2004) a consacrée à ce contour particulier :

L'examen de la littérature montre que l'intonation d'implication, à laquelle est attribuée le plus souvent une valeur assertive, est aussi très régulièrement décrite comme le marqueur privilégié des énoncés à valeur de demande de confirmation qui relèvent plutôt de la question (voir Fontaney, 1991 et Purson \& Di Cristo, 1998). (Portes 2004 : 162)

Les observations faites par Portes (2004) dans son propre corpus confirment que cet emploi est bien attesté. Ce contour est donc tout à fait adapté à notre construction, qui se présente en premier lieu, répétons-le, comme une demande de confirmation. L'exemple suivant illustre les deux emplois de l'intonation d'implication, d'abord au service d'une demande de confirmation (notre construction), sur la dernière syllabe de souffrir, puis d'une assertion, sur voir (Fig. 5) :

(24) LE BRET.

- Tu parais souffrir!

CYRANO, tressaillant, et se redressant vivement.

- Devant ce monde ?... (sa moustache se hérisse ; il poitrine.)

Moi souffrir ? ... tu vas voir ! (E. Rostand, Cyrano de Bergerac, 1898)

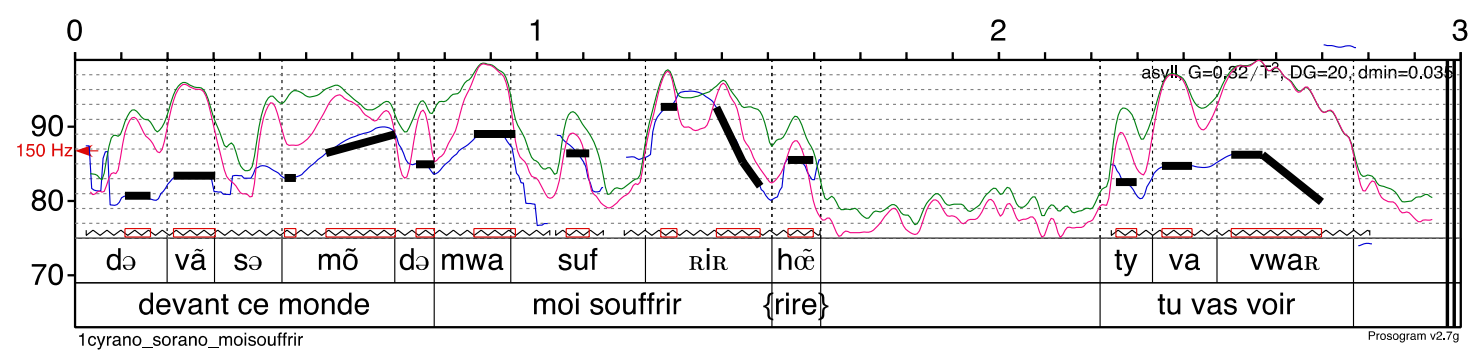

Figure 5 : prosogramme de l'exemple (24) (Daniel Sorano)

Portes (2004 : 141) mentionne encore un fait qui concerne particulièrement notre construction, « l'émergence de nombreux exemplaires de l'intonation d'implication au sein d'épisodes très clairement 
polémiques ». Dans le cas de notre structure, la demande de confirmation, plus ou moins jouée, a comme but ultime de réfuter l'idée que les contenus exprimés dans ses deux membres soient compatibles. La capacité de l'intonation d'implication à se trouver dans des contextes polémiques semble donc clairement favorable à son emploi au service de notre structure. Cette intonation paraît adaptée à la fois à sa valeur « littérale » de demande de confirmation et à sa fonction « seconde » de réfutation (v. supra, § 3.4).

En outre, il n’est probablement pas fortuit qu'un contour intonatif auquel Delattre a donné le nom d' « implication » puisse être utilisé par une structure qui fonctionne précisément par la ritualisation d’un calcul d’implicite (v. §3.3). Selon Delattre, cette intonation est utilisée pour véhiculer un sous-entendu ou afficher une certaine connivence entre le locuteur et son allocutaire. On le vérifie dans notre exemple illustré par la Fig. 5 : tout en s’opposant à l’hypothèse exprimée par son ami Le Bret (tu parais souffrir), le Cyrano joué par Daniel Sorano met prosodiquement au premier plan leur connivence, et même leur complicité, face à «ce monde » de gens hostiles (De Guiche, etc.) En témoigne encore, dans les images du film, le sourire entendu qu'il lance discrètement à son ami et dont le prosogramme (Fig. 5) traduit la manifestation sonore, juste après souffrir (1,5’').

Plus généralement, on peut penser qu'affecter notre structure d'une intonation en cloche permet, tout comme le contour montant, de traduire prosodiquement l'implicite réfutatif, mais de manière différente. L'audition des exemples de notre corpus nous incite à formuler (prudemment) l'hypothèse suivante : là où le contour montant, surtout quand il est marqué d'une amplitude importante, mime une question de dicto incrédule (<avez-vous vraiment pu dire cela, ai-je bien entendu ?> sous-entendant : <je n’en crois pas mes oreilles $>$ ), l'intonation d'implication semble affranchir notre structure de son statut originel de demande de confirmation de dicto, en faveur d'une interprétation comme question de sententia (cf. Fontaney 1991), portant non sur le dire lui-même mais sur son contenu sémantique (<pensez-vous vraiment ce que vous dites ?>), avec comme sous-entendu $<$ je ne peux pas le croire ${ }^{11}>$.

\subsubsection{Contour descendant}

Un troisième type de contour est représenté de manière nettement plus rare dans notre corpus : le contour descendant. Cette réplique de Lélio dans La Surprise de l'amour de Marivaux en donne un exemple :

(25) ARLEQUIN. - Et je remarque que vous l'aimerez aussi.

LELIO. - Moi, l’aimer ! moi, l’aimer ! (...) (Marivaux, La Surprise de l'amour, 1723)

La répétition de la structure invite l'acteur étudiant qui joue Lélio dans notre enregistrement à la réaliser avec deux contours intonatifs différents. La première occurrence de Moi, l'aimer ! est réalisée avec deux contours montants successifs sur moi et sur la syllabe finale de l'aimer, exprimant une demande de confirmation sur le mode de la surprise. La deuxième occurrence est marquée par un contour descendant, typique d'une assertion :

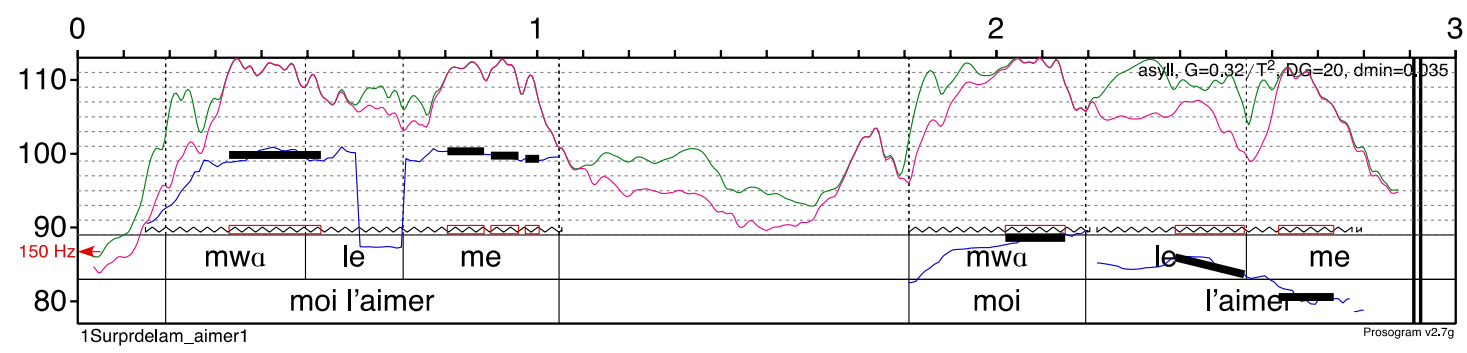

Figure 6 : prosogramme de l'exemple (25)

L'effet produit est le suivant: après s'être étonné qu’Arlequin ait pu l'associer au fait d'aimer la Comtesse, il en prend acte. Autrement dit (nous paraphrasons), il demande d'abord <avez-vous réellement émis l’idée que je pourrais aimer la Comtesse, moi ?> en sous-entendant $<$ j'en suis très surpris> puis il asserte : < oui vous émettez bien cette idée>, en entérinant le sous-entendu <je m’en désolidarise $>$. Cette intonation descendante semble bien conserver la composante échoïque de la 
structure, mais sous une forme autre qu'une demande de confirmation : c'est une sorte de mention d'une assertion de l'interlocuteur (<c'est mon interlocuteur, et pas moi, qui asserte que moi et aimer la Comtesse sont compatibles $>$ ).

Une autre occurrence d'intonation descendante affecte la structure Moi, vous venger ! dans cette réplique d’Éliante du Misanthrope :

(26) ALCESTE. - Vengez-moi de ce trait qui doit vous faire horreur.

ÉLIANTE. - Moi, vous venger ! Comment ? (Molière, Le Misanthrope, 1667)

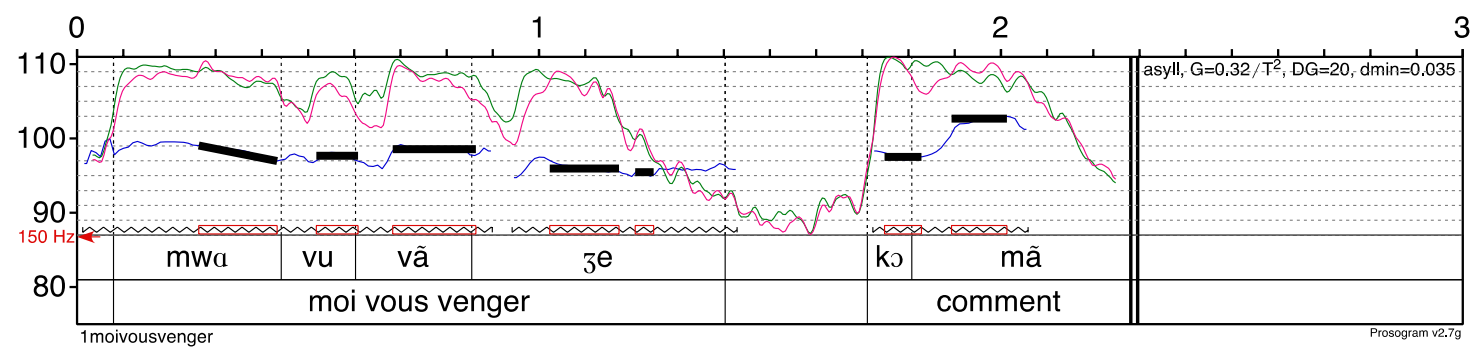

Figure 7 : prosogramme de l'exemple (26)

Elle y est suivie d'une intonation montante sur comment. Au regard de l'exemple précédent, il est intéressant de noter qu'Eliante emploie le contour descendant sans passer par l'étape de la demande de confirmation à contour montant. Elle prend acte d'emblée, assertivement, du fait qu'Alceste l'associe à sa vengeance, et elle l'interroge ensuite sur les moyens de la mettre en œuvre (<vous dites que je pourrais vous venger, je vous demande comment faire $>$ ). Evidemment, la question comment ? (sans doute rhétorique) véhicule le sous-entendu réfutatif $<$ je ne vois pas comment je pourrais vous venger $>$.

Le fait que cette réalisation intonative descendante puisse s'appliquer à notre structure est un indice que celle-ci est déjà ritualisée, et qu'elle peut s’interpréter indépendamment d'une demande de confirmation, même feinte. C'est paradoxalement avec cette intonation descendante que la construction se libère le plus de son origine interrogative, pour devenir une structure plus purement exclamative...

\section{Conclusion}

La construction exclamative que nous avons étudiée met en jeu un scénario en trois phases. L’extrait (27) peut servir à synthétiser nos observations :

(27) GeORge DAndin. - Au travers de toutes vos grimaces, j’ai vu la vérité de ce que l'on m’a dit, et le peu de respect que vous avez pour le nœud qui nous joint. Mon Dieu ! laissez là votre révérence, ce n’est pas de ces sortes de respect dont je vous parle, et vous n’avez que faire de vous moquer.

ANGELIQUE. - Moi, me moquer ! En aucune façon. (Molière, George Dandin, 1669)

D’abord, il existe (facultativement) une séquence initiatrice de la part de L1 ; ici l'accusation formulée, sous couvert d'interdiction, par Dandin : vous n'avez que faire de vous moquer. Un fait est donc validé (ou tente de l'être) dans la mémoire discursive (Angélique se moque). Ensuite, notre construction - Moi, me moquer ! - consiste en une séquence réactive de L2 (Angélique), sous la forme d'une double demande de confirmation de dicto, feinte. Le fait est ainsi remis en jeu, avec une modalité singulière : l'infinitif (le subjonctif ou le conditionnel) le transforme en une simple éventualité, qui, ainsi exhibée par un phénomène de mention, se trouve révoquée. En effet, demander confirmation de deux éléments que le discours précédent réunissait (Angélique et le fait de se moquer), revient à (feindre de) s’étonner que leur association soit seulement envisageable. Cela ouvre la voie à l'inférence négative selon laquelle cette association excède les normes admises par L2 (autrement dit, ces deux éléments sont pour elle incompatibles). Enfin, il y a facultativement une seconde séquence réactive de L2 qui consiste en une verbalisation de l'implicite négatif véhiculé par la construction (En aucune façon). 
A notre sens, l’inférence responsable de sa valeur négative s’est ritualisée sur la construction. L'examen prosodique de quelques-unes de ses occurrences théâtrales semble bien confirmer une certaine ritualisation. En effet, à côté de réalisations intonatives montantes, qui rendent bien compte de sa valeur de demande de confirmation, on a pu observer que les contours intonatifs « en cloche » et descendants semblent l'écarter de cette valeur première, en exploitant de manière différente son caractère échoïque et sa valeur réfutative. Dans une zone floue où se mêlent et se confondent interrogation et exclamation, ces réalisations prosodiques particulières, de même que certaines ponctuations, affranchissent dans une certaine mesure notre construction de son origine interrogative, pour la faire ressentir de manière plus purement exclamative.

\section{Références bibliographiques}

Akmajian, A. (1984). Sentence Types and the Form-Function Fit. Natural Language and Linguistic Theory, 1-2, 123.

Anscombre, J.-C., \& Ducrot, O. (1983). L’argumentation dans la langue. Bruxelles : Mardaga.

Boersma, P., \& Weenink, D. (1995-2015). Praat: doing phonetics by computer (version 6). En ligne: http://www.fon.hum.uva.nl/praat/

Bouveret, M. \& Legallois, D. (2012). Constructions in French. Amsterdam / Philadelphie: Benjamins.

Corminbœuf, G. \& Gachet, F. (soumis). Moi, me moquer! En aucune façon. Une construction infinitive à valeur exclamative.

Delattre, P. (1966). Les Dix Intonations de base du français. The French Review 40/1, 1-14.

Ducrot, O. (1984). Le dire et le dit. Paris : Minuit.

Fillmore, C., Kay, P. \& O’Connor, M.K. (1988). Regularity and idiomaticity in grammatical constructions: The case of let alone. Language, 64, 501-538.

Fontaney, L. (1991). À la lumière de l’intonation. La question. C. Kerbrat-Orecchioni (éd.), Lyon : PUL, 113-161.

Frantext, base textuelle, ATILF-CNRS \& Université de Lorraine. En ligne : http://www.frantext.fr/

Gachet, F. \& Corminbœuf, G. (2012). Les exclamatives de Mlle Pellaton. Actes du CMLF 2012 - $3^{\text {ème }}$ Congrès Mondial de Linguistique Française, 1743-1757. En ligne : [http://dx.doi.org/10.1051/shsconf/20120100040]

Jespersen, O. (1971=1924). La philosophie de la grammaire. Paris : Minuit.

Lambrecht, K. (1990). What, me worry? Mad Magazine sentences revisited. Proceedings of the Sixteenth Annual Meeting of the Berkeley Linguistics Society. University of California, 215-228.

Mertens, P. (2004). Le Prosogramme : une transcription semi-automatique de la prosodie. Cahiers de l'Institut de Linguistique de Louvain 30/1-3, 7-25.

Portes, C. (2004). Prosodie et économie du discours : Spécificité phonétique, écologie discursive et portée pragmatique de l'intonation d'implication. Université de Provence-Aix-Marseille I. [En ligne: https://tel.archives-ouvertes.fr/tel-00296742v2]

Wenger, N. (2009). Adult Root Infinitives. En ligne : http://satzstrukturen.de/Dateien/Wenger2009ARIs.v1.pdf

\footnotetext{
${ }^{1}$ Les occurrences de la construction collectées dans Frantext sont essentiellement datées entre le milieu du XVIIème siècle et la première moitié du XIXème siècle.

${ }^{2}$ Nous laissons de côté certaines structures apparentées, comme par exemple le cas où l'infinitif est seul : ÉGLE. - Allons, allons, tout bien examiné, mon parti est pris : donnons-nous du chagrin, séparons-nous pour deux heures, j’aime encore mieux votre cœur et son adoration que votre présence, qui m’est pourtant bien douce.

Azor. - Quoi ! nous quitter ! (Marivaux, La Dispute, 1747)
} 
${ }^{3}$ Une explication probable au choix de l'indicatif dans (9) tient à la psychologie et à la situation du personnage. Agnès est dans une position de soumission qui lui permet difficilement de s'offusquer, même lorsqu'elle est accusée par une vieille femme d'avoir blessé quelqu'un. La formule à l'infinitif serait une manière de contester qu'on puisse l'accuser ( $\approx$ «Quoi! vous osez m'associer à l'acte de blesser quelqu'un »); or Agnès n'est pas en situation de contester, et ne peut pas opérer cette généralisation par l’infinitif. En l'occurrence, elle ne fait que s'étonner naïvement d'avoir pu, dans une circonstance particulière, blesser involontairement le jeune homme qui lui fait des visites, et elle voudrait plutôt s’en excuser si le fait était avéré.

${ }^{4}$ Ce fait est considéré comme valide dans la mémoire parce qu’il a déjà été énoncé ou au moins implicité préalablement dans le discours. Dans (11) par exemple, vous blâmer fait écho à l'idée du blâme introduite dans la mémoire discursive par la séquence que tu me fasses honte des faiblesses d'un coeur dans les propos d'Euryale.

${ }^{5}$ Cette composante négative inhérente à la construction est relativement prévisible. Questionner sur un fait $p$ (validé en mémoire ou dont une tentative de validation a été réalisée) revient à orienter vers non-p. Les rapports entre interrogation et négation sont en effet bien documentés dans la littérature scientifique (Anscombre \& Ducrot 1983).

${ }^{6}$ Nous nous distançons de la position de Lambrecht (1990) qui considère que ces «réponses auto-locutées » font partie de la construction.

${ }^{7}$ Les petites majuscules notent un accent expressif. Le ‘+' note une pause.

${ }^{8}$ Dans notre construction, il y a peut-être une différence entre moi (souvent utilisé de manière « défensive » et surjouée), et lui (plus proche de la surprise authentique).

${ }^{9}$ A ce propos, parmi les biais de corpus, on peut se demander si les comédiens amateurs ont une prosodie plus naturelle ou plus stéréotypée que des acteurs plus entraînés, et si, inversement, les réalisations plus variées des acteurs chevronnés sont le résultat d'une conscience plus aiguë des possibilités prosodiques, ou au contraire d'une tendance à produire des énoncés plus artificiels. La réponse à cette question vaudrait une étude à elle seule. Sans chercher à la traiter ici, nous l'avons gardée en tête et tâché de conserver la prudence qui s’impose dans l'examen de nos données. Nous avons notamment renoncé à exploiter certaines occurrences dont les réalisations prosodiques nous semblaient trop éloignées des usages courants, paraissant vouloir frapper par leur étrangeté plutôt que par leur intelligibilité.

${ }^{10}$ Le signal sonore a été analysé sous Praat (Boersma \& Weenink 1995-2015) et la représentation graphique des tracés prosodiques a été réalisée grâce au script Prosogramme (Mertens 2004), v. Fig 1 à 7.

${ }^{11}$ Ce sous-entendu qui émerge des questions de sententia est parfois explicité dans les propos qui accompagnent la structure (v. les séquences en italiques dans les deux occurrences ci-dessous) :

- (...) Partez, ou tenez-vous caché et prêt à fuir. Je ne voudrais pas votre perte, Edmée ne la voudrait pas non plus... ainsi... Entendez-vous?

- Vous êtes insensé de croire que j'écouterai un semblable conseil. Moi, me cacher ! moi, fuir comme un coupable ! vous n’y songez pas! (Sand, Mauprat, 1852)

Moi, Guillaume Levrault, m’allier volontairement à l'aristocratie ! Moi, donner ma fille à un marquis élevé dans l'oisiveté ! Peux-tu le croire un seul instant! (Sandeau, Sacs et parchemins, 1851) 